\title{
Resonances on GaN-on-Si Epitaxies: A Source of Output Capacitance Losses in Power HEMTs
}

\author{
Mohammad Samizadeh Nikoo, Riyaz Abdul Khadar, Armin Jafari, Minghua Zhu, Student Member, IEEE, \\ and Elison Matioli, Member, IEEE
}

\begin{abstract}
GaN-on-Si high-electron-mobility transistors (HEMTs) exhibit excellent properties for efficient power conversion. Nevertheless, a considerable energy loss associated with the charging and discharging of the output capacitance (Coss) in these transistors severely limits their application at high switching frequencies. In this work we report the observation of unexpected resonances in $\mathrm{GaN}$ on-Si HEMTs. These high-frequency resonances lead to considerable energy losses in fast charging and discharging of the Coss during switching transients. We propose a simple wafer-level measurement technique to evaluate such losses at the epitaxy level, prior to the transistor fabrication. Experimental results from this technique revealed that the Silicon substrate is the main origin of these losses. Such wafer-level evaluation of Coss losses opens opportunities to characterize and optimize epitaxies for future power devices, especially those operating at high switching frequencies.
\end{abstract}

Index Terms-Output capacitance, Coss, Coss losses, EIss, GaN, HEMT, AIGaN/GaN, Si substrate.

\section{INTRODUCTION}

$\mathrm{T}$ HE-LOWER-THAN-EXPECTED efficiency of highfrequency soft-switching power converters based on $\mathrm{GaN}$ high-electron-mobility transistors (HEMTs) highlighted the energy dissipation $\left(E_{\text {DISS }}\right)$ in charging and discharging of their output capacitance $\left(C_{\text {OSs }}\right)$ [1]-[10]. The initial studies using the Sawyer-Tower (ST) technique revealed a lossy behavior in the

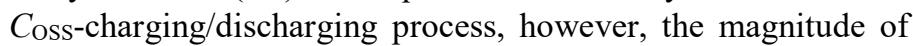
the measured losses by sinusoidal excitation was smaller than the amount of losses in real switching transients [2]. Further investigations showed that the origin of the $C_{\mathrm{OSS}}$ losses was in the epitaxial structure of power HEMTs [3]-[5], which was completely different from the previously-observed $C_{\text {oss }}$ losses in super-junction (SJ) transistors [11], [12]. A precise characterization, and ultimately reduction, of $E_{\text {DISS }}$ in power epitaxies is of great importance, especially for power devices operating at high switching-frequencies, where $C_{\text {oss }}$ losses can be even larger than conduction losses [13].

Low parasitic-capacitances of $\mathrm{GaN}$ power transistors enable ultra-fast nanosecond switching times [14], [15], resulting in extremely high-frequency harmonics, well-beyond $100 \mathrm{MHz}$. Nevertheless, practical considerations limit the frequency characterization of $C_{\text {OSS }}$ losses to typically less than 40 $\mathrm{MHz}$ [2], [7]. Packaging and external-connection parasitics are other limitations hindering the accurate measurement of $C_{\mathrm{OSS}}$ losses at very high frequencies. In this work we present a precise on-wafer measurement technique to evaluate $C_{\text {oss }}$ losses due to the epitaxial structure. This method uses a small-signal

The Authors are with the Power and Wide-band-gap Electronics Research Laboratory (POWERlab), École polytechnique fédérale de Lausanne (EPFL), CH-1015 Lausanne, Switzerland. (e-mail: elison.matioli@epfl.ch). modeling approach to evaluate large-signal $C_{\text {oss }}$ losses [8], [9], which quantifies frequency-dependent energy dissipation in the $C_{\text {Oss }}[10]$. The extremely low parasitics provided by this method enables the measurement of $C_{\text {oss-related losses at very high }}$ frequencies $>100 \mathrm{MHz}$. This provides a general characterization method of epitaxial structures, enabling their optimization prior to the transistor fabrication. This new technique revealed unexpected resonant lossy-peaks in GaN-on-Si epitaxies, which were identified to be mainly due to the Silicon substrate.

\section{Methodology}

The output capacitance of a power HEMT mainly consists of three parts: gate-drain capacitance $\left(C_{\mathrm{GD}}\right)$, drain-substrate capacitance through the vertical epitaxial structure $\left(C_{\text {epi }}\right)$, and drain-source capacitance through the two-dimensional electron gas (2DEG) $\left(C_{\text {lat }}\right)$. In power (MOS)HEMTs, $C_{\mathrm{GD}}$ is significantly smaller than the two other terms, so that one can consider $C_{\mathrm{OSS}}$ $=C_{\text {epi }}+C_{\text {lat }}$ (Fig. 1a). By applying drain-source voltage in the OFF-state, the 2DEG under the gate is depleted, resulting in a considerable reduction in $C_{\text {lat }}$ and $C_{\text {Oss. }}$. At high enough drainsource voltages, $C_{\text {oss }}$ becomes constant, approximately equal to $C_{\text {epi }}$ (Fig. 1b) [5]. $C_{\text {epi }}$ has been presented as the significant part of $C_{\text {OSs }}$ and also the main source of $C_{\text {OSs }}$ losses in power HEMTs [5]. This is a vertical capacitance sandwiched between drain pad and silicon substrate, so the capacitance mainly depends on the area of the drain pad, independent from the device layout. Although the characterization of high-frequency losses of RF epitaxies has been proposed in the literature [6], there is little knowledge on high-frequency losses of power epitaxies.

Here we present a wafer-level measurement method based on a simple proposed test structure (Fig. 2a) including a signal and ground pads to extract losses due to the epitaxial structure, which is the main source of $C_{\text {Oss }}$ losses. The signal pad dimensions should be considerably larger than thickness of the insulating epitaxy so that the effect of fringing fields can be neglected. The RF signal pad is surrounded by a considerably larger ground pad. The ground pad forms a giant capacitance $\left(C_{\infty}\right)$ with the substrate, which grounds the doped silicon
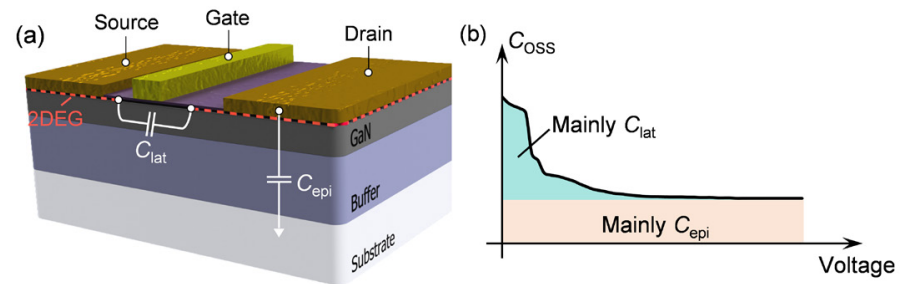

Fig. 1. (a) Simplified structure of an AIGaN/GaN HEMT showing its output capacitance $C_{\mathrm{Oss}}=C_{\mathrm{GD}}+C_{\text {epi }}+C_{\text {lat. }}$. In practice the effect of $C_{\mathrm{GD}} \ll C_{\mathrm{OSs}}$ is negligible. (b) Coss versus $V_{D S}$ curve for a commercial device showing that $C_{\text {epi }}$ dominates at high drain-source voltages. 
(a)

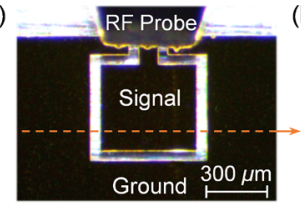

(b)

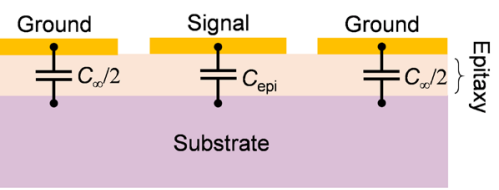

(c)

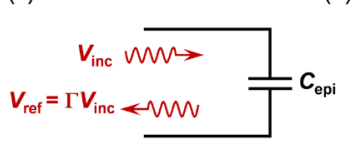

(d)
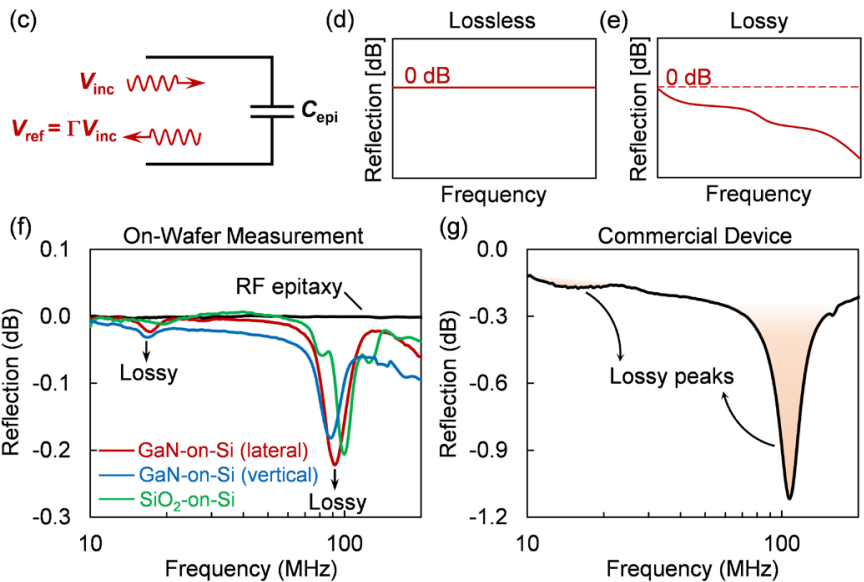

(g)

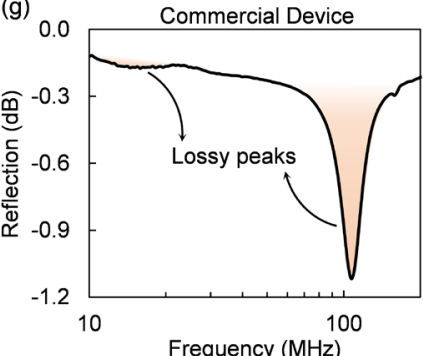

Fig. 2. (a) Optical image of the test structure measured with an RF probe. (b) Cross-section illustration of the proposed test structure. (c) Measurement method to extract losses from output capacitance to characterize epitaxial structures. Illustration of reflection coefficient for (d) lossless, and (e) lossy epitaxies. (f) Measurement results for an RF epitaxy (GaN-on-SiC), together vertical MOSFET), as well as a $\mathrm{SiO}_{2}$-on-Si test structure. (g) Measurement results for a $600-V$-rated commercial device showing similar lossy peaks. is examined with an incident wave with amplitude $V_{\text {inc }}$, and the reflection coefficient $\Gamma=V_{\text {ref }} / V_{\text {inc }}$, in which $V_{\text {ref }}$ is the reflected wave amplitude, is measured by a network analyzer (Fig. 2c). A frequency sweep of $V_{\text {inc }}$ gives full information on the behavior of the device under test (DUT), including its circuit model and losses. A lossless $C_{\text {epi }}$ results in the total reflection of the incident wave, thus $\Gamma$ is equal to $0 \mathrm{~dB}$ (Fig. 2d). Whereas a lossy $C_{\text {epi }}$ results in a partial reflection of the incident wave, thus in negative values of $\Gamma$ (Fig. 2e).

One can extract the impedance of the DUT using

$$
Z=Z_{0}(1+\Gamma) /(1-\Gamma) \text {. }
$$

where $Z_{0}=50 \Omega$ is the measurement port characteristic impedance. The quality factor $Q$ of the capacitor is obtained as

$$
Q=\operatorname{Im}(Z) / \operatorname{Re}(Z)
$$

where $\operatorname{Im}(Z)$ and $\operatorname{Re}(Z)$ represent the imaginary and real parts of $Z$, respectively. For a capacitive element, the amount of charging/discharging losses (between 0 to $V$ ) is inversely proportional to its $Q$, as

$$
E_{\text {diss }}=\frac{\pi}{2 Q} E_{\text {tot }}
$$

where $E_{\text {tot }}=1 / 2 C V^{2}$ is the total energy stored in the capacitor when charged to the voltage $V$. Thus $\pi /(2 Q)$ represents the percentage of energy losses.

Fig. 2a shows the fabricated experimental structure. The size of the signal and ground pads are $0.24 \mathrm{~mm}^{2}$ and $3.7 \mathrm{~mm}^{2}$, respectively, and the thickness of the pads is $300-\mathrm{nm}(270-\mathrm{nm}$ gold with 30-nm titanium adhesion layer). A ground-signalground (GSG) RF probe connected to a network analyzer (Keysight N5225A) was used to measure the reflection coefficient at different frequencies, with the lowest possible parasitics and an extremely high bandwidth of $50 \mathrm{GHz}$. Fig. $2 \mathrm{f}$ shows the measured reflection coefficient from test structures fabricated on four different epitaxies: GaN-on-SiC RF epitaxy, with two GaN-on-Si power epitaxies (corresponding to a lateral HEMT and a

substrate at radio frequencies (Fig. 2b). The test structure shown

GaN-on-Si power epitaxies for lateral HEMTs and vertical MOSFETs, and a test $\mathrm{SiO}_{2}$-on-Si wafer. In all cases, we fabricated devices on $2 \mathrm{~cm} \times 1.8 \mathrm{~cm}$ chips diced from 6-inch wafers. All samples on Si substrates (p-type with a resistivity of $\sim 0.02 \Omega \cdot \mathrm{cm}$ ) exhibited considerably higher losses with respect to the reference RF epitaxy, with two pronounced peaks at $\sim 17$ $\mathrm{MHz}$ and $\sim 90 \mathrm{MHz}$. The presence of lossy peaks (especially the larger one at higher frequency) for the $\mathrm{SiO}_{2}$-on-Si sample (with no $\mathrm{GaN}$ layer) suggests that that the $\mathrm{Si}$ substrate is a potential origin of $C_{\mathrm{OSS}}$ losses in GaN-on-Si HEMTs. We also observed very similar lossy peaks in some commercial GaN-on-Si power HEMTs (Fig. 2g). In this case - in an off-chip measurement we measured the reflection coefficient from drain-source of the commercial transistor, while the gate was shorted to the source. The vertical GaN-on-Si epitaxy also presented similar lossy peaks. In this case, the drift layer is lightly doped which results in a leakage current between the signal and the ground pads. This causes a lossy behavior over all frequencies and so a lower $Q$ factor. This effect can be seen in Fig. 2(f), where the vertical epitaxy (blue) shows higher background losses. Therefore, the proposed method reveals all sorts of power dissipations including resonant and leakage losses.

\section{Results}

We used the proposed method to quantify the amount of output capacitance losses due to these resonances for epitaxies with different buffer thicknesses. Using (1) and (3), we extracted the capacitance as well as the losses related to $C_{\mathrm{epi}}$, for two GaN-on-Si power epitaxies 4- $\mu \mathrm{m}$ (Fig. 3a) and 5- $\mu \mathrm{m}$ (Fig. 3b) buffer thicknesses (similar to the lateral GaN-on-Si epitaxy shown in Fig. 2(f)). There are two distinct peaks corresponding to losses for both epitaxies. Exactly at these peaks, abrupt changes in capacitance are observed, which shows a resonance happening in the epitaxial structure. Although the second epitaxy is $25 \%$ thicker than the first one, the resonance frequencies are very close, showing a material dependency of such resonances. As expected, the thicker buffer has a lower capacitance and so a lower stored energy while the dominant part of losses due to the substrate is about the same. This results in a higher relative losses for the thicker epitaxy.

Fig. 3 shows the importance of characterizing $C_{\text {oss }}$ losses at
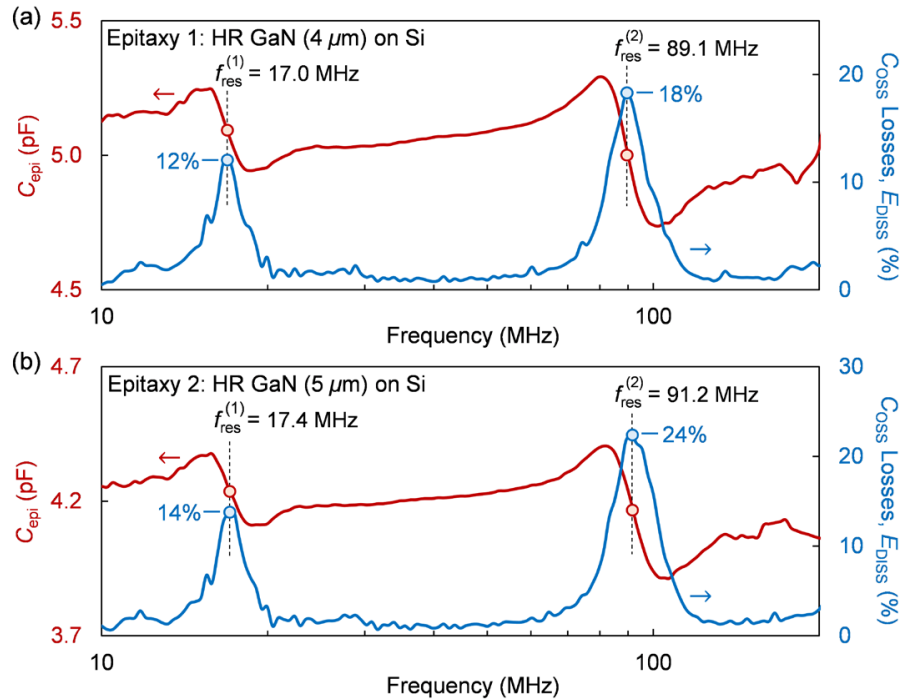

Fig. 3. Extracted capacitance and losses of the test capacitor on (a) 4- $\mu \mathrm{m}$ thick, and (b) 5- $\mu \mathrm{m}$-thick carbon-doped high-resistive (HR) GaN buffers. In both cases, there are lossy resonances at $\sim 17 \mathrm{MHz}$, and $\sim 90 \mathrm{MHz}$.

om IEEE must be obtained for all other uses, in any current or future media, including reprinting/republishing this material for advertising or promotional purposes, creating new collective works, for resale or redistribution to servers or lists, or reuse of any copyrighted component of this work in other works. 
(a)

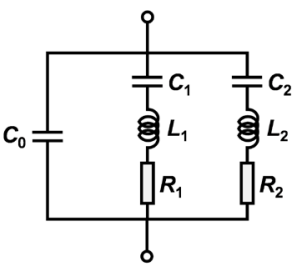

(c)

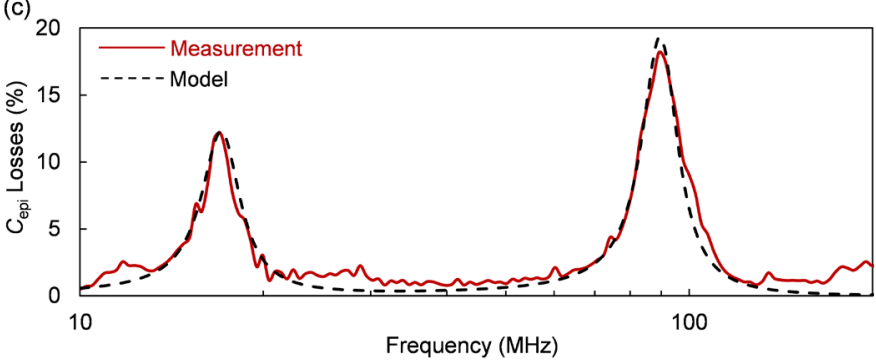

Fig. 4. (a) Proposed circuit model for output capacitance losses, together with (b) circuit parameters of the 4- $\mu$ m-thick buffer sample. (c) Measured (solid line) and modeled (dashed line) $C_{\text {epi }}$ losses.

high frequencies, since a low frequency test, for instance up to $10 \mathrm{MHz}$, would not show such significant frequency-dependent losses happening at high frequencies. This could be a potential reason of higher measured losses with pulse excitation (containing higher harmonics) in comparison to sine-wave in ST method [2]. For instance, at $10-\mathrm{MHz}$, the employed pulse waveform in [2] had a 5-ns rise-time, and the $C_{\text {oss }}$ losses corresponding to this waveform was not limited to the fundamental frequency of $10-\mathrm{MHz}$, but it included all the frequency content of the pulse, exceeding 100-MHz. This also shows that single-tone ST measurements, previously performed up to $35 \mathrm{MHz}$ [2], [13], should be performed at much higher frequencies to cover the typical switching harmonics of wideband-gap transistors. In addition, Fig. 3 shows a 1-2\% loss over a wide frequency range, which indicates the ability of the proposed method in capturing background $C_{\text {epi }}$ losses, caused by other phenomena such as the resistivity of substrate.

A circuit model of the $C_{\text {epi }}$ can be extracted from the results shown in Fig. 3. The model includes a lossless capacitor $C_{0}$ in parallel with two lossy $R L C$ resonant branches (Fig. 4a). Fig. 4b shows the extracted circuit parameters for the 4- $\mu \mathrm{m}$-thick buffer. Fig. $4 \mathrm{c}$ presents the measured and modeled $C_{\text {epi }}$ power dissipation, showing a good agreement between them. We employed the model presented in Fig. $4 \mathrm{a}$ in a circuit-level simulation with LTspice to extract the amount of $C_{\text {Oss }}$ losses due to these resonances for different switching times. Fig. 5a shows the power dissipated in $R_{1}$ and $R_{2}$ (Fig. 4a) representing the low-frequency and high-frequency resonances. The switching time and voltage are $2-\mathrm{ns}$ and $400-\mathrm{V}$, respectively. The results for 5-ns switching at the same switching voltage level are shown in Fig. 5b. As expected, the high-frequency resonance leads to much higher losses for the faster switching transient. The low-frequency resonance, however, is considerably slower than the typical switching transient of a GaN transistor, so both switching times of 2-ns and 5-ns lead to an almost equal power loss. It should be noted that the power loss continues happening after the switching transient is finished. For example for low-frequency resonance, there is a considerable power loss even 100-ns after the switching transient. This cannot be observed in the previous simplified models of output capacitance losses [4], since they do not show any resonances. Fig. $5 \mathrm{c}$ shows the $C_{\text {oss-related energy }}$ (C) 2021 IEEE. Personal use of this material is permitted. Permission from IEEE must be obtained for all other uses, in any current or future media,
including reprinting/republishing this material for advertising or promotional purposes, creating new collective works, for resale or redistribution to servers or lists, or reuse of any copyrighted component of this work in other works.
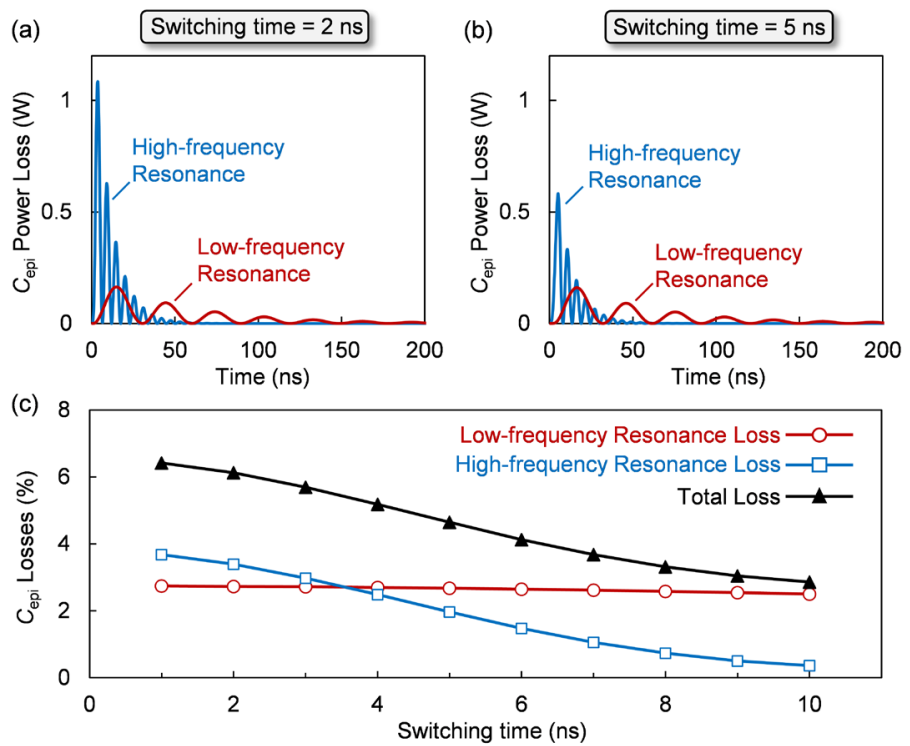

Fig. 5. Simulated Coss power losses of the 4- $\mu$ m-thick buffer sample for 400-V (a) 2-ns, and (b) 5-ns switching transients. The plots show separately the power loss representative for the low- and high-frequencies resonates. (c) Extracted Coss energy dissipation corresponding to the low- and highfrequencies resonates, as well as the total loss.

dissipation versus switching time for $400-\mathrm{V}$ switching, from 1 to $10 \mathrm{~ns}$, as well as the share of each resonance in the total loss. For fast switching transients, about $6 \%$ of the stored energy is dissipated during charging and discharging. This number is higher for the $5-\mu \mathrm{m}$-thick buffer and can reach to about $8 \%$ of the stored energy, showing that $C_{\text {oss }}$ losses can be potentially more severe for devices with a higher blocking voltage capability.

\section{CONCLUSION}

We proposed a new on-wafer test method to characterize

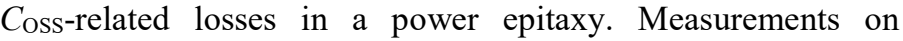
different power epitaxies revealed new insights on $C_{\mathrm{Oss}}$-related losses. Two lossy resonances at $17 \mathrm{MHz}$ and $90 \mathrm{MHz}$ were observed, which are of very different nature from previously proposed models and opens opportunities for further investigations. The proposed method can be used to evaluate power electronic devices operating at high switching frequencies.

\section{REFERENCES}

[1] K. Surakitbovorn and J. Rivas-Davila, "Evaluation of GaN transistor losses at $\mathrm{MHz}$ frequencies in soft switching converters," in Proc. IEEE 18th Workshop Control Modelling Power Electron. (COMPEL), Jul. 2017. doi: 10.1109/COMPEL.2017.8013330.

[2] G. Zulauf, S. Park, W. Liang, K. Surakitbovorn, and J. Rivas-Davila, " $C_{\text {oss }}$ losses in $600 \mathrm{~V}$ GaN power semiconductors in soft-switched, high- and very-high-frequency power converters," IEEE Trans. Power Electron., vol. 33, no. 12, pp. 10748-10763, Dec. 2018. doi: 10.1109/TPEL.2018.2800533.

[3] M. Guacci, M. Heller, D. Neumayr, D. Bortis, J. W. Kolar, G. Deboy, C. Ostermaier, and O. Häberlen, "On the origin of the $C_{\text {oss-losses in soft }}$ switching GaN-on-Si power HEMTs," IEEE J. Emerg. Sel. Topics Power Electron., vol. 7, no. 2, pp. 679-694, Jun. 2019. doi: 10.1109/JESTPE.2018.2885442.

[4] J. Zhuang, G. Zulauf, J. Roig, J. D. Plummer, and J. Rivas-Davila, "An Investigation into the Causes of Coss Losses in GaN-on-Si HEMTs," in Proc. IEEE 20th Workshop Control Modelling Power Electron. (COMPEL), Jun. 2019. doi: 10.1109/COMPEL.2019.8769661. 5] J. Zhuang, G. Zulauf, and Juan Rivas-Davila, "Substrate bias effect on e-
mode GaN-on-Si HEMT Coss losses," in Proc. IEEE 19th Workshop mode GaN-on-Si HEMT Coss losses," in Proc. IEEE 19th Workshop and optimized power wafers at the epitaxial level for future 
Control Modelling Power Electron. (COMPEL), Jun. 2018. doi: 10.1109/WiPDA.2018.8569205.

[6] H. Chandrasekar, M. J. Uren, M. A. Casbon, H. Hirshy, A. Eblabla, K. Elgaid, J. W. Pomeroy, P. J. Tasker, and M. Kuball, "Quantifying Temperature-Dependent Substrate Loss in GaN-on-Si RF Technology," IEEE Trans. Electron Devices, vol. 66, no. 4, pp. 1681-1687, April 2019. doi: 10.1109/TED.2019.2896156.

[7] M. Samizadeh Nikoo, A. Jafari, N. Perera, and E. Matioli, "Measurement of large-signal output capacitance of transistors based on nonlinear resonance," IEEE Trans. Power Electron., vol. 35, no. 3, pp. 2242-2246, Mar. 2020. doi: 10.1109/TPEL.2019.2938922.

[8] M. Samizadeh Nikoo, A. Jafari, N. Perera, and E. Matioli, "New insights on output capacitance losses in wide-band-gap transistors," IEEE Trans. Power Electron., vol. 35, no. 7, pp. 6663-6667, Jul. 2020. doi: 10.1109/TPEL.2019.2958000.

[9] M. Samizadeh Nikoo, A. Jafari, N. Perera, and E. Matioli, "Output capacitance losses in wide-band-gap transistors: a small-signal modeling approach," 2020 Int. Symp. On Power Semiconductor Devices and ICs, Sep. 2020, pp. 190-193. doi: 10.1109/ISPSD46842.2020.9170093.

[10] N. Perera, A. Jafari, L. Nela, G. Kampitsis, M. Samizadeh Nikoo, and E. Matioli, "Output-capacitance hysteresis losses of field-effect transistors," in Proc. IEEE 21th Workshop Control Modelling Power Electron. (COMPEL), Nov. 2020. doi: 10.1109/COMPEL49091.2020.9265823.
[11] J. Roig and F. Bauwens, "Origin of anomalous $C_{\text {Oss }}$ hysteresis in resonant converters with superjunction FETs," IEEE Trans. Electron Devices, vol. 62, no. 9, pp. 3092-3094, Sep. 2015. doi: 10.1109/TED.2015.2455072.

[12] M. Kasper, R. M. Burkart, G. Deboy, and J. W. Kolar, "ZVS of power MOSFETs revisited," IEEE Trans. Power Electron., vol. 31, no. 12, pp. 8063-8067, Dec. 2016. doi: 10.1109/TPEL.2016.2574998.

[13] G. Zulauf, Z. Tong, J. D. Plummer, and J. M. Rivas-Davila, "Active power device selection in high- and very-high-frequency power converters," IEEE Trans. Power Electron., vol. 34, no. 7, pp. 6818-6833, Jul. 2019. doi: 10.1109/TPEL.2018.2874420.

[14] S. Moench, P. Hillenbrand, P. Hengel, and I. Kallfass, "Pulsed measurement of sub-nanosecond $1000 \mathrm{~V} / \mathrm{ns}$ switching $600 \mathrm{~V}$ GaN HEMTs using 1.5 GHz low-impedance voltage probe and $50 \mathrm{Ohm}$ scope," in Proc. IEEE 5th Workshop Wide Bandgap Power Devices Appl. (WiPDA), Oct./Nov. 2017, pp. 132-137. doi: 10.1109/WiPDA.2017.8170535.

[15] J. W. Simonaitis, B. Slayton, Y. Yang-Keathley, P. D. Keathley, and K. K. Berggren, "Precise, sub-nanosecond, and high-voltage switching of complex loads enabled by gallium nitride electronics," arXiv:2102.03271, 2021. 